\title{
Risk Factors of Anastomotic Thrombosis in 200 Head and Neck Free Flaps among Asian Patients
}

\author{
Takaya Makiguchi $^{1}$, Kazunobu Hashikawa ${ }^{1}$, Daisuke Sugiyama ${ }^{2}$, Satoshi Yokoo ${ }^{3}$, \\ Hiroto Terashi $^{1}$, Kenichi Nibu ${ }^{4}$, Shunichi Kumagai ${ }^{2}$, Shinya Tahara ${ }^{1}$ \\ ${ }^{1}$ Department of Plastic Surgery, Kobe University Graduate School of Medicine, Kobe, Japan \\ ${ }^{2}$ Department of Evidence Based Laboratory Medicine, Kobe University Graduate School of Medicine, Kobe, Japan \\ ${ }^{3}$ Department of Stomatology and Oral Surgery, Gunma University Graduate School of Medicine, Gunma, Japan \\ ${ }^{4}$ Department of Otolaryngology-Head and Neck Surgery, Kobe University Graduate School of Medicine, Kobe, Japan \\ Email: takayamakiguchi@gmail.com
}

Received February 6, 2012; revised April 15, 2012; accepted April 27, 2012

\begin{abstract}
Background: Despite excellent results from free flap transfers, postoperative thrombosis does occur and, although infrequent, causes total loss of the flap. We investigate the risk factors of anastomotic thrombosis in head and neck free flaps. Study Design, Subjects and Methods: A retrospective analysis was carried out on 200 consecutive free flaps applied to patients who underwent head and neck reconstruction. Multiple logistic regression analysis was conducted to identify risk factors of anastomotic thrombosis, taking into account twelve factors: diabetes, obesity, hypertension, hypertriglyceridemia, proteinuria, age, gender, smoking habit, habitual alcohol intake, previous chemotherapy and radiation, and hypercholesterolemia. Results: Hypertension, female gender, aging, and drinking habit were statistically significant risk factors of postoperative thrombosis. Conclusions: Our data suggest that four factors mentioned above are possible predictors of anastomotic thrombosis in free flaps used for head and neck.
\end{abstract}

Keywords: Free Flap; Anastomotic Thrombosis; Risk Factor

\section{Introduction}

Free flap transfers have proved highly effective in the reconstruction of complex defects in the head and neck region [1-7]. Despite excellent results, postoperative thrombosis does occur and, although infrequent, causes total loss of the flap. To increase the success rate of free flap transfers, it is essential to evaluate the factors affecting anastomotic thrombosis.

Kinked pedicle vessels or compression may be one of risk factors of thrombosis. We have, however, often encountered cases which pedicle vessel had not been apparently kinked and had not been compressed by large hematoma at re-explored operation. In these cases other factors must have affected thrombosis. Previous reports had mixed factors of patients and intraoperative variables. Few have reported in detail on risk factors affecting anastomotic thrombosis focused on demographic and clinical variables of patients in head and neck reconstruction among Asian people.

In this study we focused on demographic and clinical variables of patients, so didn't include intraoperative variables such as ischemia time, surgery time, blood transfusion, vessels choices and so on. This study analyzes 200 consecutive free flaps used in the reconstruction of head and neck defects in patients most of whom had undergone reconstruction after cancer resection.

\section{Patients and Methods}

Records of 200 consecutive patients (144 men and 56 women, between 16 and 82 years old; median 62 years; all of Asian origin) who had undergone head and neck reconstructive surgery with free flap transfers at Kobe University Hospital between June 2002 and January 2007, were analyzed. We collected all data set from computerized microsurgery database of our department recorded with FileMaker Pro $^{\circledR}$ and medical records. This study was performed after approval by the Ethics Committee of Kobe University Graduate School of Medicine. Vein anastomoses were sutured either end-to-end or end-to-side with nylon thread, and artery anastomoses were sutured end-to-end. Twelve factors were analyzed to determine their significance on anastomotic thrombosis: diabetes, obesity, hypertension, hypertriglyceridemia, proteinuria, age, gender, smoking habit, habitual alcohol intake, previous chemotherapy and radiation, and hypercholesterolemia. The definition of the respective factors is shown in Table 1. Patients with a body mass index (BMI) of over 25 were classified as obese, because patients with a BMI 
Table 1. The twelve factors and their significance in anastomotic thrombosis.

\begin{tabular}{ll}
\hline \#Obesity & \#Aging \\
$(\mathrm{BMI} \geq 25)$ & $(\geq 70$ years old $)$ \\
\#Diabetes & \#Sex (female) \\
(HbA1c $\geq 5.8$, or $\mathrm{FBS} \geq 110 \mathrm{mg} / \mathrm{dL}$, or treatment of & \#Hypercholesterolemia \\
previously diagnosed type2 diabetes not including type1) & $(\geq 220 \mathrm{mg} / \mathrm{dL}$, specific treatment of this lipid abnormality) \\
\#Hypertension & \#Radiation \\
$\quad$ (SBP $\geq 140 \mathrm{mmHg}$ or DBP $\geq 90 \mathrm{mmHg}$ or treatment of & \#Chemotherapy \\
previously diagnosed hypertension) & \#Smoking habit \\
\#Hypertriglyceridemia & included all smokers except those quitting smoking since more than \\
$(\geq 150 \mathrm{mg} / \mathrm{dL}$ or specific treatment for this lipid abnormality) & a year ago \\
\#Proteinuremia & \#Habitual alcohol intake \\
& more than once a week \\
\hline
\end{tabular}

of over 30 (considered excessive for Asians) were only two ( $1 \%$ of all patients). The ratio of obesity was smaller than that in the general population of Japan because a large number of patients with malignancies were included. Renal disorders associated with vascular disease have been reported in a large population-based study [8]. We included proteinuria as one of data subset. We defined drinking habit as intake of alcohol more than once a week. Our definition of smoking habit included all smokers except those quitting smoking since more than a year ago. Aging people are defined as those over than seventy years old. The donor sites were the radial forearm (93/200: 47\%), the rectus abdominis muscle $(51 / 200: 26 \%)$, the jejunum (45/200: $23 \%)$, the latissimus dorsi (7/200: $4 \%$ ), osteocutaneous scapular (3/200: $1.5 \%)$ and tensor fascia lata (1/200: $0.5 \%)$.

The flaps were designed to avoid vein grafts; only one patient needed a vein graft, and none of the patients required multiple simultaneous free flaps. A microvascular fellow took progressive responsibility for free flap transfer over several years training period.

Of the 200 patients $91 \%$ (181/200) underwent reconstruction after cancer resection and the rest after release of cervical contracture, cranial reconstruction and eye socket surgery. All of patients were Asian people.

Logistic regression model for multivariate analysis with stepwise procedure was carried out to identify possible risk factors (among those mentioned above) for anastomotic thrombosis. A p value of $<0.05$ was considered statistically significant. R version 2.21 (R Development Core Team) was used for all statistical analyses.

\section{Results}

Two hundred free flaps were transferred to head and neck defects in 200 consecutive patients. The number of patients, their respective risk factors and the thrombosis rate are shown in Table 2. Thrombosis at the anastomotic site occurred in $7.5 \%(15 / 200)$ of the flaps: at the venous site in 7 patients, at the arterial site in 4 and at both the arterial and the venous sites in 4 (Table 3), and in $74 \%$ $(11 / 15)$ of the flaps within 3 days of surgery (Table 4). The thrombosis was re-explored in 13 of these 15 patients,
Table 2. Patient number and ratio, and factors related to anastomotic thrombosis.

\begin{tabular}{cll}
\hline Factor & Number (percentage) & $\begin{array}{c}\text { Number of thromboses } \\
\text { (percentage) }\end{array}$ \\
\hline Obesity (BMI $\geq 25)$ & $23(11.5 \%)$ & $4(17.4 \%)$ \\
Diabetes & $31(15.5 \%)$ & $1(3.2 \%)$ \\
Hypertension & $49(24.5 \%)$ & $7(14.3 \%)$ \\
Hypertriglyceridemia & $30(15.0 \%)$ & $2(6.7 \%)$ \\
Proteinuremia & $17(8.5 \%)$ & $2(11.8 \%)$ \\
Age $(\geq 70)$ & $47(23.5 \%)$ & $6(12.8 \%)$ \\
Sex (female) & $56(28.0 \%)$ & $8(14.3 \%)$ \\
Hypercholesterolemia & $40(20.0 \%)$ & $6(15.0 \%)$ \\
Radiation & $27(13.5 \%)$ & $3(11.1 \%)$ \\
Chemotherapy & $43(21.5 \%)$ & $6(14.0 \%)$ \\
Smoking habit & $120(60.0 \%)$ & $8(6.7 \%)$ \\
Habitual alcohol intake & $131(65.5 \%)$ & $13(9.9 \%)$ \\
\hline
\end{tabular}

Table 3. Vessel of thrombosis.

\begin{tabular}{cc}
\hline Site of thrombosis & Number (percentage) \\
\hline Vein & $7(47 \%)$ \\
Artery & $4(27 \%)$ \\
Vein and artery & $4(27 \%)$ \\
Total & 15 \\
\hline
\end{tabular}

Table 4. Time of thrombosis.

\begin{tabular}{cc}
\hline Postoperative days & Total $(\mathrm{n}=15)$ \\
\hline Within 24 hrs & $7(47 \%)$ \\
$2-3$ days & $4(27 \%)$ \\
After 3 days & $4(27 \%)$ \\
\hline
\end{tabular}

and thrombectomy was not feasible in the remaining 2 . The overall salvage rate was $54 \%(7 / 13)$. There was no case in which pedicle vessel was apparently kinked and was compressed by large hematoma at re-explored operation. Total flap necrosis occurred in 8 flaps; therefore, the overall success rate of free-flap reconstruction of head and neck defects was 96 percent.

Four significant risk factors for postoperative thrombosis at the anastomotic site were identified after multivariate analysis: hypertension (odds ratio $4.45 ; 95 \% \mathrm{CI}=$ 
1.30 to 15.40 ), age (odds ratio $5.49 ; 95 \% \mathrm{CI}=1.49$ to 20.24 ), the female gender (odds ratio $4.45 ; 95 \% \mathrm{CI}=1.54$ to 19.48), and habitual alcohol intake (odds ratio 9.21; $95 \% \mathrm{CI}=1.64$ to 51.67 ) (Table 5). The thrombosis rate in obese patients was more than double that in non-obese patients (Table 2). Among obese patients with complications of anastomotic thrombosis, three quarters were women, and half of obese patients with thrombosis had hypertension. Multivariate analysis with stepwise procedure showed that among the twelve factors, the female gender, hypertension, drinking habit and aging were significant predictors of thrombosis, obesity was not.

\section{Discussion}

The purpose of this study was to review predictors of postoperative thrombosis at anastomotic sites of free flap transfers in the head and neck region. The female gender, aging, hypertension, and habitual alcohol intake may be risk factors for anastomotic thrombosis; diabetes, obesity, proteinuria, hypertriglyceridemia, hypercholesterolemia, previous irradiation and chemotherapy, and smoking habit, had no significant effect. In this study, regions of free tissue transfers were limited to the head and neck, and most of the patients had undergone cancer resection.

The female gender was a predictor of postoperative thrombosis, whereas no other study has reported any association between the female gender and anastomotic thrombosis in free flaps. Numerous studies have shown that women on hemodialysis have a significantly low arterial-venous fistula (AVF) patency [9]. The incidence of thrombosis in the hepatic artery in liver transplantation is higher in women than in men, one possible explanation being that women have smaller vessels and lower arterial flow than men, although opinions vary [10].

Also, few other studies have reported an association between aging, hypertension or habitual alcohol intake and anastomotic thrombosis in free flap transfers, and not many assessments have been made of the risk factors of postoperative thrombosis in head and neck free flap surgery $[7,11]$. The mechanism of how aging, habitual alcohol intake and hypertension increase postoperative thrombosis is not known; however, these three are risk factors of vascular disease, such as arteriosclerosis, and may influence vessels at donor or recipient sites. Hyper- tension is generally thought to affect arterial lesion more than venous, as it is closely relevant to arteriosclerosis. When we investigated risk factors of anastomotic thromboses only in venous thromboses ( 7 cases of this study; Table 3), female and drinking habit were signify- cant factors. Accordingly hypertension and aging, that to say, the other two significant factors of four mentioned above may possible to be actually risk factors of only arterial thromboses. Because we define 'habitual drinking' as occasional drinking, such as on weekends, further study is needed with measured daily volume. Nonetheless, we assume that non-drinkers have a lower rate of postoperative thrombosis.

We must closely observe Asian patients with these four predictors undergoing free flap transfers for head and neck reconstruction, although further study is needed for understanding the mechanism of how these predictors increase such postoperative thrombosis.

Diabetes, obesity, smoking habit, previous irradiation and chemotherapy, proteinuria, hypertriglyceridemia, and hypercholesterolemia had no significant effect on anastomotic thrombosis.

Several experimental studies have suggested an association between noncontrolled hyperglycemia and vessel thrombosis [12]. We believe that perioperative management of hyperglycemia is crucial; otherwise, the incidence of anastomotic thrombosis could be higher, as observed in experimental models. The level of blood sugar is to some degree commonly controlled perioperatively. In clinical practice, diabetes shows no apparent increased incidence of thrombosis [4,13]. Similarly, our results revealed no relation between postoperative thrombosis and diabetic patients.

Smoking was not a risk factor for anastomotic thrombosis, and our findings are supported by a number of studies [1,5-7]. Controversially Haughey et al reported that cigarette smoking during 2 weeks before surgery is a significant risk factor for flap complications, which included not only anastomotic thrombosis but also wound dehiscence infection, hematoma, fistula and so on [3]. Nahabedian reported smoking more than 10 cigarettes per day was associated with flap failure [4]. The definition of smoking habit might contribute to differences in results of these other studies. Ours include all smokers

Table 5. Multiple logistic regression analysis of factors associated with anastomotic thrombosis.

\begin{tabular}{|c|c|c|c|c|c|c|}
\hline Factors & Coefficient & Standard error & Odds ratio & $95 \% \mathrm{CI}$ & Wald chi-squared & $\mathrm{P}$ value \\
\hline Hypertension & 1.49 & 0.63 & 4.45 & $1.30-15.30$ & 5.62 & 0.02 \\
\hline Female & 1.70 & 0.65 & 5.47 & $1.54-19.49$ & 6.90 & 0.01 \\
\hline Age $\geq 70$ years old & 1.70 & 0.67 & 5.49 & $1.49-20.25$ & 6.55 & 0.01 \\
\hline Habitual alcohol intake & 2.22 & 0.88 & 9.21 & $1.64-51.67$ & 6.37 & 0.01 \\
\hline
\end{tabular}


except patients stopping smoking for a long time before operation. Smoking until just before surgery or perioperatively might affect the thrombosis rate. Results also may be affected by amount of cigarettes a day. Smoking habit may affect other flap complication except anastomotic thrombosis such as fistula formation, wound dehiscence, infection or hematoma. We think further studies are needed on a larger number of patients and on identifying differences in amount of cigarettes and when they smoke. Our and others' findings indicate, however, that patients forgoing smoking only perioperatively and for some period before surgery should not be denied free flap transfers [1,5-7].

Previous radiation therapy was also not a significant predictor of thrombosis. The effects of radiation on vessels, such as impairment of endothelium-dependent vasodilation, perivascular fibrosis, endothelial damage and microvascular occlusion, can decrease the quality of recipient vessels [14,15]. Histological changes have been observed in only the artery, not the vein [14]. Radiation probably has a smaller effect on vein than on artery thrombosis. In our study, anastomotic thrombosis of the vein was about two-fold that of the artery; thus, no relation was observed between anastomotic thrombosis and previous radiotherapy. Further studies for identifying the difference between arterial and venous thrombosis are needed. Singh et al. reported that multivariate analysis revealed prior radiation exposure was a significant predictor of recipient cite complication, which included not only anastomotic thrombosis but also infection, wound dehiscence, fistula formation, hematoma and so on [1]. Our study, however, focused on only anastomotic thrombosis. This might contribute to differences in results. Prior radiation might increase other flap complication such as fistula formation, or wound dehiscence or partial flap loss. As in our study, numerous clinical studies have demonstrated that previous radiotherapy of the head and neck does not affect flap survival or anastomotic patency [4-6,16].

Obesity was statistically not a predictor of postoperative thrombosis in our study, and as shown in another study [13]. No other studies have evaluated obesity and postoperative anastomotic thrombosis restricted to the head and neck region. Our result showed that obese patients had a higher rate of thrombosis than non-obese patients. But among obese patients with complications of anastomotic thrombosis, three quarters were women, and half of obese patients with thrombosis had hypertension. Multivariate analysis with a stepwise procedure showed that the female gender, hypertension, aging and drinking habit were significant predictors of thrombosis, obesity was not. That obesity was not a risk factor for anastomotic thrombosis in head and neck free flaps is a conclusion that cannot be drawn from this study alone. Since many patients with malignant tumors were included in this survey, we were not able to evaluate the risk of intrinsic obesity. Obesity may be a predictor of thrombosis in free tissue transfers in extremities or the trunk, or in external injuries and benign disorders. We don't know how generalizable our result would be for more obese than our patient population.

\section{Conclusion}

Our data suggest that the female gender, aging, hypertension, and habitual alcohol intake may be possible predictors of anastomotic thrombosis in free flaps used for head and neck reconstruction. We think further studies are needed on a larger number of patients with external injuries and benign disorders and on identifying differences in patients population most of who were with cancer such as this study and more obese patients population. Our study suggests that attention should be paid to obese women patients and to obese patients with hypertension. Further studies are needed on a larger number of patients and on identifying differences between arterial and venous thrombosis, as well as on free flap sites other than the head and neck region.

\section{Acknowledgements}

The Corresponding Author had full access to all the data in the study and takes responsibility for the integrity of the data and the accuracy of the data analysis.

\section{REFERENCES}

[1] B. Singh, P. G. Cordeiro, E. Santamaria, et al., "Factors Associated with Complications in Microvascular Reconstruction of Head and Neck Defects," Plastic and Reconstructive Surgery, Vol. 103, No. 2, 1999, pp. 403-411. doi:10.1097/00006534-199902000-00007

[2] T. Nakatsuka, K. Harii, H. Asato, et al., "Analytic Review of 2372 Free Flap Transfers for Head and Neck Reconstruction Following Cancer Resection," Journal of Reconstructive Microsurgery, Vol. 19, No. 6, 2003, pp. 363-368. doi: $10.1055 / \mathrm{s}-2003-42630$

[3] B. H. Haughey, E. Wilson, L. Kluwe, et al., "Free Flap Reconstruction of the Head and Neck: Analysis of 241 Cases," Otolaryngology Head and Neck Surgery, Vol. 125, No. 1, 2001, pp. 10-17. doi:10.1067/mhn.2001.116788

[4] M. Y. Nahabedian, N. Singh, E. G. Deune, et al., "Recipient Vessel Analysis for Microvascular Reconstruction of the Head and Neck," Annals of Plastic Surgery, Vol. 52, No. 2, 2004, pp. 148-155. doi:10.1097/01.sap.0000095409.32437.d4

[5] K. Bozikov and Z. M. Arnez, "Factors Predicting Free Flap Complications in Head and Neck Reconstruction July," Journal of Plastic, Reconstructive \& Aesthetic Surgery, Vol. 59, No. 7, 2006, pp. 737-742. doi:10.1016/j.bjps.2005.11.013

[6] J. D. Suh, J. A. Sercarz, E. Abemayor, et al., "Analysis of 
Outcome and Complications in 400 Cases of Microvascular Head and Neck Reconstruction," Archives of Otolaryngology, Vol. 130, No. 8, 2004, pp. 962-966. doi:10.1001/archotol.130.8.962

[7] K. H. Simpson, P. G. Murphy, P. M. Hopkins, et al., "Prediction of Outcomes in 150 Patients Having Microvascular Free Tissue Transfers to the Head and Neck," British Journal of Plastic Surgery, Vol. 49, No. 5, 1996, pp. 267-273. doi:10.1016/S0007-1226(96)90154-X

[8] L. Palaniappan, M. Carnethan and S. P. Formann, "Association between Microalubuminuria and Metabolic Syndrome: NHANES III," American Journal of Hypertension," Vol. 16, No. 11, 2003, pp. 952-958. doi:10.1016/S0895-7061(03)01009-4

[9] L. Vernaglione, G. Mele, C. Cristofano, et al., "Comorbid Conditions and Gender Impact the Primary Survival of Distal Radio-Cephalic Arteriovenous Fistula Inpatients on Long-Term Hemodialysis," Journal of Nephrology, Vol. 18, No. 3, 2005, pp. 276-281.

[10] T. Ikegami, Y. Hashikura, Y. Nakazawa, et al., "Risk Factors Contributing to Hepatic Artery Thrombosis Following Living-Donor Liver Transplantation," Journal of HepatoBiliary-Pancreatic Surgery, Vol. 13, No. 2, 2006, pp. 105109. doi:10.1007/s00534-005-1015-y

[11] S. C. Bonawitz, R. H. Schnarrs, A. I. Rosenthal, et al., "FreeTissue Transfer in Elderly Patients," Plastic and Reconstructive Surgery, Vol. 87, No. 6, 1991, pp. 1074-1079. doi:10.1097/00006534-199106000-00009

[12] L. B. Colen, A. Stevenson, V. Sidorov, et al., "Microvascular Anastomotic Thrombosis in Experimental Diabetes MelliTus," Plastic and Reconstructive Surgery, Vol. 99, No. 1, 1997, pp. 156-162. doi:10.1097/00006534-199701000-00024

[13] R. K. Khouri, B. C. Cooley, A. R. Kunselman, et al., "A Prospective Study of Microvascular Free-Flap Surgery and Outcome," Plastic and Reconstructive Surgery, Vol. 102, No. 3, 1998, pp. 711-721. doi:10.1097/00006534-199809030-00015

[14] S. Schultz-Mosgau, G. G. Grabenbauer, F. Wehrhan, et al., "Histomorphological Structural Changes of Head and Neck Blood Vessels after Preor Postoperative Radiotherapy," Strahlentherapie und Onkologie, Vol. 178, No. 6, 2002, pp. 299-306.

[15] J. A. Beckman, A. Thakore, B. H. Kalinowski, et al., "Radiation Therapy Impairs Endothelium-Dependent Vasodilation in Humans," Journal of the American College of Cardiology, Vol. 37, No. 3, 2001, pp. 761-765. doi:10.1016/S0735-1097(00)01190-6

[16] B. P. Bengston, M. A. Shusterman, B. J. Baldwin, et al., "Influence of Prior Radiotherapy on the Development of Postoperative Complications and Success of Free Tissue Transfers in Head and Neck Reconstruction," American Journal of Surgery, Vol. 166, No. 4, 1993, pp. 326-330. doi:10.1016/S0002-9610(05)80325-3 